\title{
Structural Vaccinology for Viral Vaccine Design
}

\author{
Mohd Ishtiaq Anasir and Chit Laa Poh* \\ Centre for Virus and Vaccine Research, Sunway University, Bandar Sunway, Malaysia
}

Although vaccines have proven pivotal against arrays of infectious viral diseases, there are still no effective vaccines against many viruses. New structural insights into the viral envelope, protein conformation, and antigenic epitopes can guide the design of novel vaccines against challenging viruses such as human immunodeficiency virus (HIV), hepatitis $C$ virus, enterovirus $A 71$, and dengue virus. Recent studies demonstrated that applications of this structural information can solve some of the vaccine conundrums. This review focuses on recent advances in structure-based vaccine design, or structural vaccinology, for novel and innovative viral vaccine design.

\section{OPEN ACCESS}

Edited by:

Douglas Paul Gladue,

Plum Island Animal Disease Center

USDA ARS, United States

Reviewed by:

Rino Rappuoli,

GlaxoSmithKline, Italy

Chad Edward Mire,

The University of Texas Medical Branch at Galveston, United States Yongqun $\mathrm{He}$,

University of Michigan Health System, United States

*Correspondence: Chit Laa Poh

pohcl@sunway.edu.my

Specialty section:

This article was submitted to

Virology,

a section of the journal

Frontiers in Microbiology

Received: 23 November 2018 Accepted: 25 March 2019

Published: 16 April 2019

Citation: Anasir MI and Poh CL (2019) Structural Vaccinology for Viral Vaccine Design.

Front. Microbiol. 10:738. doi: 10.3389/fmicb.2019.00738
Keywords: structural vaccinology, vaccine design, protein structure, epitope, virus

\section{INTRODUCTION}

Vaccination has considerably reduced morbidity and mortality caused by viruses such as smallpox virus and poliovirus. However, there remain many viruses without effective vaccines including dengue virus, human immunodeficiency virus (HIV), enterovirus A71, and hepatitis C virus. In addition, emerging infectious diseases will continue to threaten public health. Therefore, rapid vaccine development is mandatory to prevent pandemics. Currently, there are only 17 licensed vaccines in the United States against 13 viruses. These vaccines are either live-attenuated vaccines (11 vaccines), whole-inactivated vaccines (4 vaccines), or virus-like particle vaccines (2 vaccines) (Graham and Sullivan, 2018). Notably, the inactivated and live-attenuated vaccines are based on technologies developed decades ago. Taking live-attenuated vaccines as an example, the development of this type of vaccine is empirical and time-consuming. The virulent virus will be passaged in either tissue cultures or live animals until the avirulent form was isolated. In the settings of challenging viruses, these traditional approaches have failed to produce effective vaccines. Additionally, traditional approaches would not be swift enough to respond to epidemics caused by emerging viruses. This is due to lack of knowledge and experience with viral pathogenesis, viral growth, and attenuating determinants of the emerging viruses (Graham and Sullivan, 2018). Therefore, the focus for vaccine development against challenging viruses should be based on newer rational vaccine design platforms. In this article, we will cover recent advances in the development of novel vaccines facilitated by structural vaccinology. Additionally, we will discuss how structural vaccinology could lead to the discovery of antibodies for passive immunization. Finally, we will provide suggestions of how structural vaccinology can be exploited for future vaccine design.

\section{STRUCTURAL VACCINOLOGY APPROACH}

Structural biology approaches have enabled the determination of the structure of whole viruses, viral proteins, and antigen-antibody complexes (Kaufmann et al., 2010; Lee and Wilson, 2015; Anasir et al., 2017a,b). The three-dimensional (3D) structures 
provide crucial insights into the tertiary structure and position of the viral epitopes. Ultimately, the detailed structural information can be exploited to solve some of the challenging problems impeding vaccine development against viruses. Structural vaccinology is a rational approach to generate an effective vaccine that involves (1) determining the atomic structure of the antigen or antigen-antibody complex, (2) remodeling the antigen or the epitope by reverse molecular engineering, (3) incorporating the re-engineered antigen or epitope into one of the vaccine platforms, and (4) testing the safety and efficacy of the candidate vaccine in vivo (Figure 1).

In the first step of this approach, the $3 \mathrm{D}$ structure of the antigen or antigen-antibody complex will be determined using either one or more of the structural biology tools such as X-ray crystallography, cryo-electron microscopy, and nuclear magnetic resonance. Thereafter, the antigen will be remodeled based on the structural information of the antigen. In addition, the immunological and bioinformatics knowledge should be synergistically combined with the structural insights to design an effective antigen. In most cases, the re-engineering process involves mutations of certain amino acids to stabilize immunogenic conformations, mask unwanted non-neutralizing antibody epitopes, optimize antigen thermostability, or introduce additional epitopes on the antigen. Next, the re-engineered antigen could be incorporated into one of the vaccine platforms such as the recombinant protein vaccine, the viruslike particle vaccine, or the live-attenuated vaccine. Finally, the immunogenicity and efficacy of the candidate vaccine should be tested in animal models including mice, ferrets, and macaques. Based on the preclinical findings, the candidate vaccine could be redesigned to improve its immunogenicity and efficacy. Structural vaccinology was shown to be successful in overcoming the challenges facing the bacterial vaccine field. For instance, the 3D structures of H-binding protein of Men B and the pili of group B Streptococcus have facilitated the design of improved vaccines (Van Regenmortel, 2016). Similarly, structural vaccinology has been used to improve the efficacy of viral vaccines.

\section{HUMAN IMMUNODEFICIENCY VIRUS}

Human immunodeficiency virus is a serious public health threat with more than 35 million people living with HIV/AIDS worldwide in 2017. To date, there are no effective vaccines or drugs against HIV. Major barriers to vaccine development include the extreme diversity of HIV strains and the high malleability of its envelope. Due to these barriers, protective immune responses against HIV tend to be polyclonal and involve antibodies directed to a plethora of epitopes. Having structural information for the variable antigens in the viral envelopes is essential to design a vaccine that can elicit potent broadly neutralizing antibodies. HIV is one of the most studied pathogens in the context of structural vaccinology application. However, the development of an effective vaccine against HIV through a structural vaccinology approach remains elusive.

\section{Modification of Envelope Glycoprotein gp120}

The glycoprotein (gp) 120 that is present on the surface of the HIV envelope has been identified as the main target for neutralizing antibodies (Pantophlet and Burton, 2006). However, its variability and malleable nature contribute to host immune evasion (Chen et al., 2009). Several groups have reported the development of structure-based vaccine candidates based on the modified gp120. For instance, structure-based modifications of gp120 significantly stabilized its core domain at the co-receptor binding site (Dey et al., 2009). Subsequent immunization studies revealed that the conformational stabilization was associated with increased humoral immune responses targeting the stabilized site. Although the initial modifications were unsuccessful to produce an effective vaccine against HIV, the study initiated the screening for broadly neutralizing antibodies against reengineered gp120 proteins (Wu et al., 2010; Huang et al., 2018).

Another major barrier to HIV vaccine development is the induction of non-neutralizing antibodies by numerous epitopes on its envelope (McGuire et al., 2014). Such antibodies could hinder the function of its broadly neutralizing counterparts. The V3 loop of gp120 is an example of a non-neutralizing antibody epitope. Previous studies revealed that V3 immunogenicity could be diminished by introducing a hydrophobic amino acid substitution (A316W mutation) to reduce its conformational flexibility (de Taeye et al., 2015). Recently, two hydrophobic mutations, S306L and R308L, were found to further stabilize the V3 region, potentially by maintaining the burial of the V3 region in the pocket formed by V1/V2 domains (de Taeye et al., 2018). Binding studies showed that these hydrophobic mutations impaired non-neutralizing antibody interactions with the V3 loop. Immunization studies in rabbits revealed that the stabilization of the V3 region was associated with reduced induction of non-neutralizing antibodies, while the induction of neutralizing antibodies remained the same. These data demonstrated that structural vaccinology could be exploited to reduce the induction of non-neutralizing antibodies.

\section{Stabilization of Prefusion-Closed HIV Envelope}

Human immunodeficiency virus envelope in its prefusion-closed state is the main target of broadly neutralizing antibodies (Wang et al., 2016; Ozorowski et al., 2017). Due to the extreme diversity of HIV strains, sequential immunization with HIV envelopes adopting the prefusion-closed conformation from different strains might be needed to induce sufficiently broadly neutralizing antibodies. Despite many attempts to stabilize the HIV envelope in its prefusion-closed state, these variants were found to fluctuate between closed and open prefusion states (Ozorowski et al., 2017). Recently, Rutten et al. (2018) described a universal approach to produce high-quality prefusion-closed HIV envelopes through a repair-and-stabilize approach (Rutten et al., 2018). In the repair stage, they substituted rare strain-specific residues of the envelopes with more prevalent residues. In the stabilization stage, regions crucial for envelope refolding were modified to increase stability. One of the variants 
A Basic methods and tools for structural vaccinology

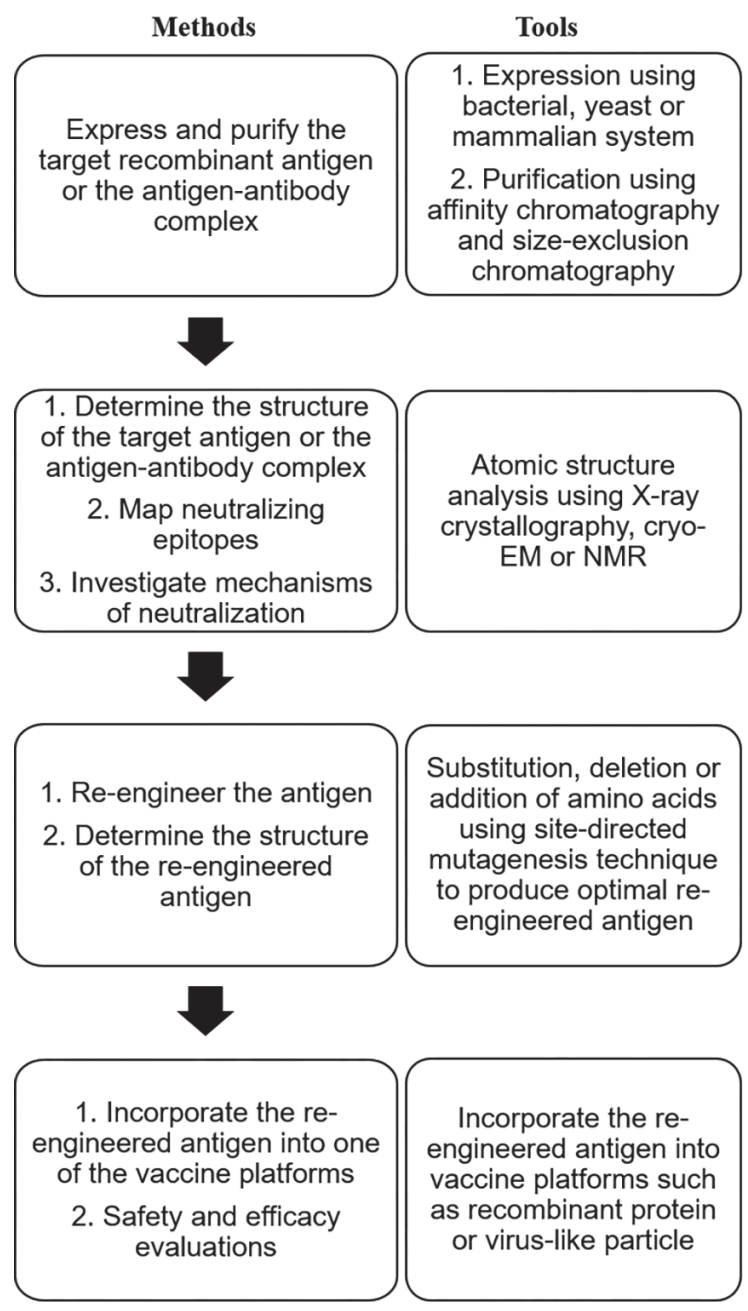

B Structural vaccinology approach

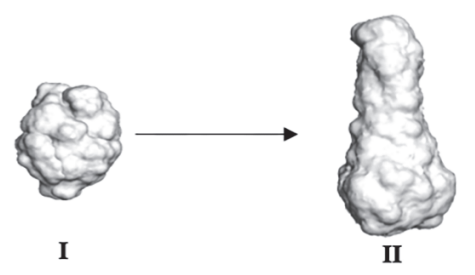

Spontaneous irreversible conformational change from immunogenic (I) to non-immunogenic (II)

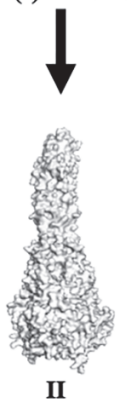

Determination of the antigen structure (II)

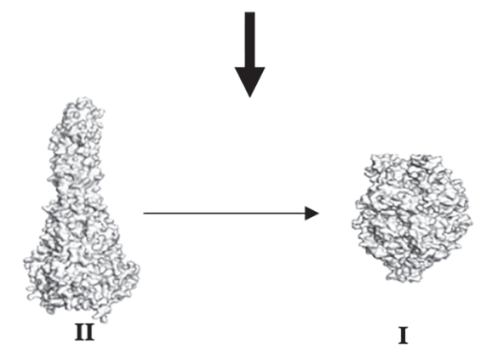

Re-engineer the antigen (II) to lock the immunogenic conformation (I)

\section{$\downarrow \uparrow$}

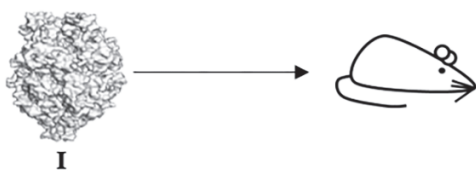

Safety and efficacy testing of the antigen (I) in animal models

FIGURE 1 | Structural vaccinology for vaccine design. (A) Basic methods and tools for structural vaccinology. (B) Irreversible conformational change (immunogenic to non-immunogenic) of viral antigens occurred spontaneously during recombinant antigen expression. To overcome this problem, the three-dimensional structure of the antigen (II) will be determined using structural biology techniques such as X-ray crystallography, cryo-electron microscopy (cryo-EM), and nuclear magnetic resonance (NMR). Based on the structure, the antigen will be re-engineered to produce an optimal antigen adopting a specific immunogenic conformation (I). Next, the re-engineered antigen could be incorporated into one of the vaccine platforms such as the recombinant protein vaccine platform. Thereafter, the safety and efficacy of the candidate vaccine should be tested in animal models. The antigen could be re-designed based on the animal testing evaluations to improve its immunogenicity and efficacy. RSV F glycoprotein was used as an illustrative example. PDB IDs: 4MMU and 3RKI.

designated as ConC_base0, which carried seven stabilizing substitutions, was found to be stable at $37^{\circ} \mathrm{C}$ for 4 weeks. This variant exhibited increased binding toward broadly neutralizing antibodies along with reduced affinity toward non-neutralizing antibodies. Detailed inspection of the variant structure revealed that the inter- and intra-protomer stabilizing mutations provided synergistic effect to stabilize the envelope in the prefusion-closed structure. Hence, this approach may be a better strategy to generate high-quality prefusion-closed HIV envelopes for future development of efficacious vaccines against HIV. 


\section{Monoclonal Antibodies for Passive Immunization Against HIV}

The screening of broadly neutralizing antibodies against HIV was initiated by the structural determination of the gp120-CD4 receptor complex (Kwong et al., 1998). Earlier, the attempts to generate broadly neutralizing antibodies specifically directed toward the CD4 binding site were unsuccessful. The failure was partly due to the fact that the gp120 used in the screening was reactive with many other antibodies, including the nonneutralizing antibodies. To generate specific antibodies targeting the CD4 binding site, gp120 was modified to eliminate the other antigenic regions while preserving the CD4 binding site (Wu et al., 2010). One of the variants designated as resurfaced stabilized core 3 (RSC3) was chosen to be used in the screening of broadly neutralizing monoclonal antibodies. From the screening, three monoclonal antibodies, which are VRC01, VRC02, and VRC03, were found to bind strongly and specifically to RSC3. VRC01 displayed impressive breadth of reactivity by neutralizing 91\% of the 190 circulating HIV-1 genetic subtypes. Subsequent structural studies revealed that VRC01 engaged the CD4 binding site of gp120 in a similar manner to the CD4-gp120 interactions (Zhou et al., 2010). Currently, VRC01 is in the phase II clinical trial as a passive immunization strategy (Huang et al., 2018).

\section{HEPATITIS C VIRUS}

Hepatitis C virus is the leading cause of liver diseases such as cirrhosis, liver failure, and liver cancer. More than 64 million people are chronically infected with hepatitis C virus (Manns et al., 2017). Liver transplantation is the main treatment undertaken for those with cirrhosis and liver cancer (Manns et al., 2017). However, the treatment does not fully eliminate the virus, resulting in possible viral rebound and infection of the transplanted liver (Manns et al., 2017). High cost is also associated with other antiviral treatments (Cox, 2015), underscoring the importance of developing a vaccine against hepatitis $\mathrm{C}$ virus.

\section{Epitope-Based Vaccines Against Hepatitis C Virus}

Similar to HIV, the development of a vaccine against hepatitis $\mathrm{C}$ virus has been hampered by the variability and diversity of target antigens. E2 glycoprotein is the main target for hepatitis $\mathrm{C}$ vaccine development. To date, the structure of full-length E2 has yet to be determined (Pierce et al., 2017). Importantly, two crystallographic structures of the engineered E2 core from two genotypes are available (Kong et al., 2013; Khan et al., 2014). In addition, various structures of hepatitis $C$ viral antigens in complexes with broadly neutralizing antibodies have been determined (Kong et al., 2012a,b, 2015; Deng et al., 2013; Krey et al., 2013; Meola et al., 2015). These studies identified multiple epitopes that could elicit broadly neutralizing antibodies. One of the epitopes, designated as epitope I, is located near the $\mathrm{N}$-terminus of E2. The epitope was found to elicit arrays of human (Keck et al., 2013) and murine antibodies (Tarr et al., 2006). Structural studies revealed that epitope I adopted a $\beta$-hairpin fold when bound to neutralizing antibodies (Kong et al., 2012b; Sandomenico et al., 2016).

Initially, a cyclic variant of epitope I adopting the $\beta$-hairpin fold was designed to generate and characterize monoclonal antibodies specific against the epitope variant (Sandomenico et al., 2016). This antigen was able to elicit antibodies in mice. However, the antibodies lacked neutralization activity when tested. Subsequently, Pierce et al. (2017) designed cyclic antigens to stably present epitope I in its antibody-bound conformation using the natural scaffold approach (Pierce et al., 2017). They identified $\theta$-defensin protein, which adopted the $\beta$-hairpin substructure nearly identical to the antibody-bound epitope I structure (Conibear et al., 2013). Based on the cyclic $\theta$-defensin structure, they designed two cyclic epitope I antigens comprising minimal scaffolded structures of the epitope. Immunogenicity studies revealed that the cyclic antigens induced higher epitopespecific serum responses in comparison to the linear peptide. Subsequent viral neutralization studies indicated that the cyclic antigens were superior immunogens than the linear epitope. However, the antibody responses elicited by the cyclic antigens were still low. Various options are available to improve cyclic epitope 1 immunogenicity. For instance, the cyclic antigens could be displayed on a virus-like particle or other nanoparticles to enhance immune responses against them.

In another approach, Pierce et al. (2017) designed a bivalent truncated E2 antigen displaying two copies of epitope I on its surface. By analyzing E2 core structures, they identified a site suitable to display the second epitope I. However, the bivalent antigen elicited lower neutralizing antibody responses than its monovalent counterpart. This indicated that further understanding of the host humoral responses against E2 is required for successful vaccine design in the future.

\section{RESPIRATORY SYNCYTIAL VIRUS}

Respiratory syncytial virus (RSV) is an etiological agent that causes lower respiratory tract infection in infants and children and often leads to hospitalizations. Previously, a formaldehydeinactivated RSV vaccine candidate was found to enhance the disease upon subsequent infection with the wild-type virus, while live-attenuated vaccine development has yet to unearth an acceptable balance of RSV attenuation (Moghaddam et al., 2006).

\section{Prefusion F Stabilization}

F glycoprotein is an attractive antigen for vaccine development as it contains multiple neutralizing epitopes. The protein forms a lollipop-shaped structure with its C-terminus inserted into the virion membrane. During viral entry, it rearranges from a metastable prefusion state to a highly stable postfusion state (Figure 2). Previous studies revealed that the metastable prefusion state accounted for most of the neutralizing activity (Magro et al., 2012). Moreover, McLellan et al. (2013b) identified an antigenic site $\varnothing$ that was bound by several prefusion F-specific antibodies. The antigenic site $\varnothing$ is located at the top of the prefusion $\mathrm{F}$ trimer and is readily accessible by the antibodies even on the crowded virion surface. The prefusion $\mathrm{F}$ was also 


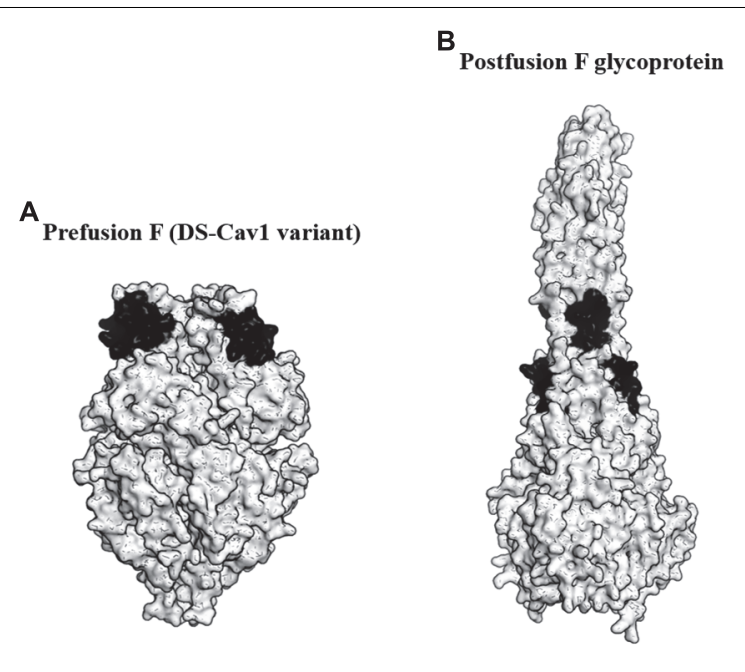

FIGURE 2 | Targeting the prefusion F glycoprotein of respiratory syncytial virus (RSV). (A) Surface representation of the DS-Cav1 F glycoprotein variant (PDB ID: 4MMU). DS-Cav1 adopted the prefusion F glycoprotein conformation. The antigenic site $\varnothing$ is shown in black. (B) Surface representation of the trimeric postfusion F glycoprotein (PDB ID: 3RKI). The disrupted antigenic site $\varnothing$ caused by structural rearrangement from the prefusion to postfusion $\mathrm{F}$ is shown in black.

found to be the target of RSV-neutralizing activity in human sera. Importantly, the antigenic site $\varnothing$ is only present on prefusion $\mathrm{F}$ and not on the postfusion F antigen (Steff et al., 2017).

To identify the prefusion $\mathrm{F}$ with the highest stability at the antigenic site $\varnothing$, McLellan et al. (2013a) produced over 100 variants of the protein. These variants possessed mutations either to stabilize the antigenic site $\varnothing$ or to destabilize the postfusion F. From the study, they identified a variant favoring the prefusion conformational state designated as DS-Cav1. DS-Cav1 carried S155C-S209C double mutations to form a disulfide bond and cavity-filling mutations (S190F and V207L). Studies in mice, macaques, and calves revealed that DS-Cav1 elicited potent neutralizing responses (McLellan et al., 2013a; Steff et al., 2017). Crucially, the studies using calves concluded that the use of DSCav1 was able to extend the duration of protection conferred by maternal immunization, potentially lowering RSV disease burden in infants (Steff et al., 2017). In 2017, DS-Cav1 entered phase I clinical trial and the trial is currently ongoing.

\section{INFLUENZA A VIRUS}

Influenza causes significant disease and death in the human population especially among the young and elderly (Shao et al., 2017). Currently, individuals are vaccinated with seasonal influenza vaccine that stimulates humoral immune responses toward the globular head domain of the viral hemagglutinin (HA). HA is a glycoprotein that acts as a mediator of viral entry into host cells (Sautto et al., 2018). One major problem with the current vaccine is that the antibodies elicited are highly strain specific, as the globular head domain is not conserved across strains. Therefore, the vaccines must be reformulated annually based on the anticipated influenza strain. Furthermore, most influenza vaccines are produced in an egg-based production system. Previous study showed that this production system caused amino acid substitutions on the HA during passaging and led to reduction in vaccine efficacy (Wu et al., 2017). In addition, the production process is slow and can cause severe production bottleneck during an epidemic.

\section{Universal Influenza Vaccine}

Many investigators characterized antibody-HA complexes to elucidate the host humoral immune responses against influenza A. Corti et al. (2011) identified a broad neutralizing antibody targeting the $\mathrm{F}$ subdomain within the highly conserved stem domain of HA. Subsequent comprehensive sequence analysis revealed a high degree of conservation within the stem domain, unlike the variable head domain (Mallajosyula et al., 2015). More recently, evolutionary analysis indicated that the stem domain evolved slowly over time than the evolution of the head domain (Kirkpatrick et al., 2018). In addition, the study found that the stem evolution did not aid in the host immune evasion. Thus, universal vaccine development against influenza virus strains should focus on the $\mathrm{HA}$ stem region instead of the head region.

To develop a universal influenza vaccine, Krammer et al. (2013) designed multiple chimeric HA (cHA) constructs combining the stem domain from the $\mathrm{H} 1$ strain with the unique head domain derived from various strains. They tested the protective potential of the cHA constructs against multiple challenge viruses (Hai et al., 2012; Krammer et al., 2013). Sequential vaccination with the cHA constructs in mice elicited stem-specific polyclonal humoral responses. The vaccination also protected mice against heterologous and heterosubtypic influenza virus challenges (Hai et al., 2012). Subsequent studies using female ferrets revealed that the vaccination with cHA reduced viral titers in nasal turbinates, lungs, and olfactory bulbs (Krammer et al., 2014). Recently, a confirmatory study was performed in male ferrets. It was found that live-attenuated vaccines expressing cHAs were indeed able to confer protection during virus challenge (Nachbagauer et al., 2018).

In a second approach, another group designed immunogens comprising only the stem region designated as mini-HAs (Impagliazzo et al., 2015). They constructed multiple mini-HAs that were stable as either monomers or trimers. The mini-HAs were found to induce high titers of antibodies binding to the HA of group 1 (H1, H5, and H9) and group 2 (H3 and H7) influenza A strains. The trimeric mini-HAs displayed full protection of mice against heterosubtypic influenza challenge. In contrast, the monomeric mini-HAs only conferred partial protection. Consistently, monkeys vaccinated with the trimeric mini-HA had significantly reduced fever after being challenged with the H1N1 virus. Many influenza vaccines based on the cHA and headless HA approaches are currently undergoing clinical trials (WHO). For instance, in an ongoing phase I clinical trial, adults aged from 18 to 39 were given a live-attenuated influenza vaccine expressing cHA followed by an inactivated vaccine expressing another cHA (NIH, United States). 


\section{Multidomain Antibody to Confer Protection Against Influenza Infection}

Due to the conservation of the HA stem region, many groups are actively developing antibodies directed toward the stem domain (Krammer et al., 2014; He et al., 2015). The stem-specific antibodies displayed broader specificities than the antibodies directed toward the HA head domain (Joyce et al., 2016). Although several of these antibodies have entered clinical trials, their use as prophylactic agents remained elusive due to incomplete coverage of the various influenza strains.

Laursen et al. (2018) utilized structure-based antibody design with the aim to develop an antibody that could provide broad and long-lasting protection against influenza A and B strains. They designed a multidomain antibody designated as MD3606 comprising four antibodies linked to a human IgG1 Fc. The four antibodies were SD36, SD38, SD83, and SD84. SD36 was found to neutralize influenza A virus group 2, while SD38 was found to neutralize both group 1 and group 2. Additionally, SD84 and SD83 were found to neutralize all influenza B strains. Structural analysis of these antibodies revealed that these antibodies were able to bind to highly conserved epitopes. When administered intravenously or expressed locally by a viral vector, MD3606 was found to confer broad protection in mice against influenza $\mathrm{A}$ and B viral infections. Further development of MD3606 is warranted as it has the potential to confer passive protection throughout the influenza season, provided the preclinical findings could be replicated in clinical trials.

\section{DENGUE VIRUS}

In recent decades, dengue virus (DENV) has emerged as a significant public health threat with an estimated 390 million infections annually (Bhatt et al., 2013). Dengue infections are caused by four antigenically distinct serotypes (DENV14) that can cross-react with each other immunologically. Currently, there is only one recombinant live-attenuated vaccine (Dengvaxia) approved for dengue prevention in endemic populations (Guy et al., 2015). However, the vaccine has low efficacy (53\%) for seronegative individuals (Hadinegoro et al., 2015). Dengvaxia is also associated with increased risk of hospitalization for dengue among vaccinated children from 2 to 5 years of age (Hadinegoro et al., 2015). A recent clinical trial concluded that Dengvaxia led to increased risk of antibodydependent enhancement (ADE) in the seronegative individuals (Halstead, 2017). ADE is a phenomenon in which the elicited antibodies during primary infection are cross-reactive with the heterologous serotypes but are non-neutralizing. The antibodybound virus is more easily internalized by $\mathrm{Fc} \gamma$ receptor-positive myeloid cells, leading to infection enhancement. Thus, it is desirable for a vaccine to induce only neutralizing antibodies but not non-neutralizing antibodies. In the case of dengue infection, $\mathrm{ADE}$ has been identified as the central mechanism associated with dengue hemorrhagic fever and dengue shock syndrome during secondary infections (Guzman et al., 2013). Thus, the success of dengue vaccine design hinges on the elimination of $\mathrm{ADE}$ in addition to balanced immune responses against all four serotypes.

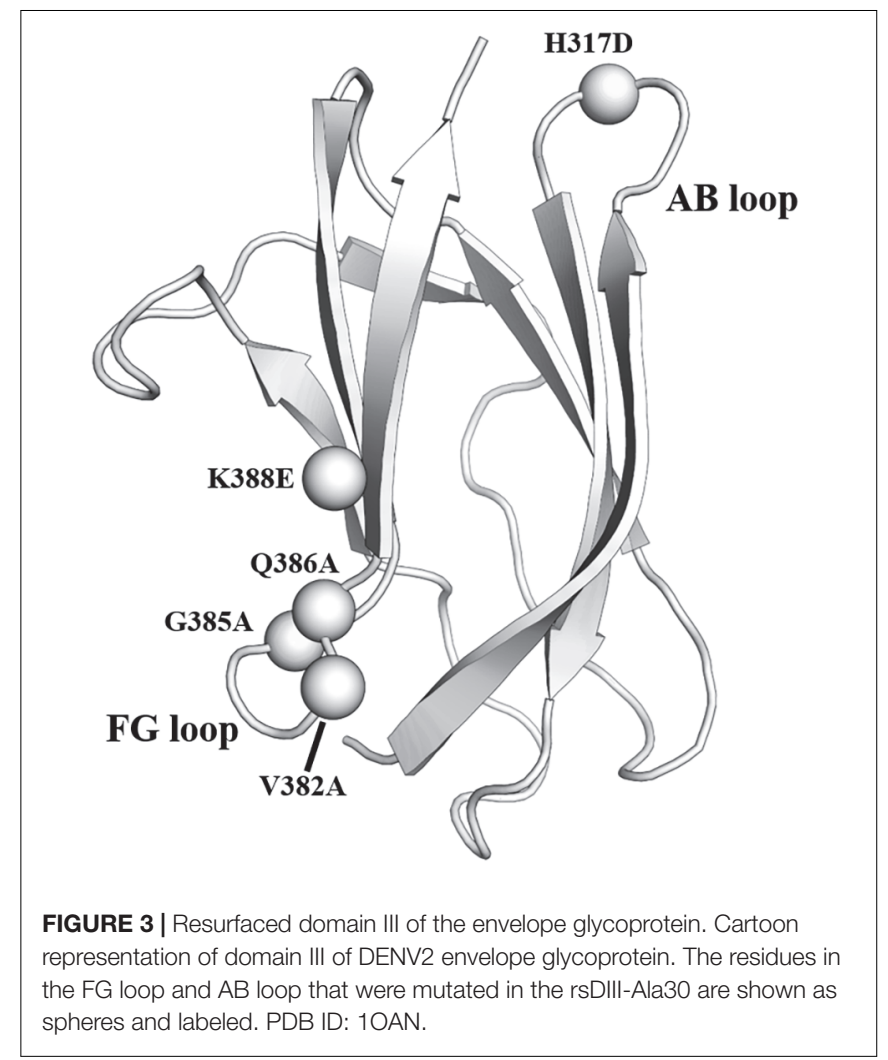

\section{Masking of Non-neutralizing Epitopes}

The mature virion of DENV is coated by glycoprotein E. It contains three domains, which are domain I (EDI), domain II (EDII), and domain III (EDIII) (Kuhn et al., 2002). EDIII is an attractive antigen as it is targeted by broadly neutralizing antibodies. However, previous animal studies revealed that although immunization using EDIII proteins induced robust antibody responses, neutralization was modest and limited to serotype-specific responses (Hermida et al., 2006; Saejung et al., 2007; Zhang et al., 2007; Batra et al., 2010). The presence of non-neutralizing antibodies targeting the immunodominant epitopes likely accounted for the limited neutralization potential of EDIII protein (Frei et al., 2018). To address the issue, a panel of resurfaced EDIII proteins was designed. Non-neutralizing epitopes such as the $\mathrm{AB}$ loop epitope and the serotype-specific FG loop epitope were masked in these variants (Frei et al., 2018). One of the resurfaced EDIII designated as rsDIII-Ala30 was found to only bind to the neutralizing antibodies $4 \mathrm{E} 11$ and $4 \mathrm{E} 5 \mathrm{~A}$, but not the non-neutralizing antibody $(2 \mathrm{H} 12)$ and the serotype-specific antibody (3H5) (Figure 3). Upon immunization in mice, rsDIIIAla30 elicited broadly reactive and cross-neutralizing antibody responses against DENV1-3. However, its antisera are insufficient to confer protection from DENV2 infection in mice.

\section{Structure-Based Anti-dengue Antibody Design}

Previously, antibody 4E11 was identified to neutralize dengue viruses of all serotypes (Thullier et al., 1999, 2001; Cockburn 
et al., 2012). However, its neutralizing activity was limited, potentially due to epitope inaccessibility on the mature virion (Tharakaraman et al., 2013). To improve the neutralizing potential of 4E11, Tharakaraman et al. (2013) produced a $4 \mathrm{E} 11$ variant designated as $4 \mathrm{E} 5 \mathrm{~A}$ that carried five amino acid substitutions. 4E5A showed marked improvement in affinity toward EDIII of DENV4. However, the affinity of 4E5A toward DENV4 is modest in comparison to its affinities to other DENV serotypes. Subsequently, they performed structural analysis to design an antibody designated as Ab513 (Robinson et al., 2015). This antibody differed from the starting murine antibody $4 \mathrm{E} 11$ through (1) six point mutations and deletion at position 26 to improve affinity toward DENVs and (2) mutations to humanize the antibody. Challenge studies revealed that the mice administered with Ab513 had accelerated recovery of platelet level post-DENV challenge. Furthermore, the passively immunized mice displayed significant reduction in the viral load upon challenge. Currently, Ab513 is in the preclinical trial to determine the optimal dosing scheme for use as a therapeutic or prophylactic antibody against DENV infection.

\section{ENTEROVIRUSES CAUSING HAND, FOOT, AND MOUTH DISEASE}

Hand, foot, and mouth disease (HFMD) is a significant public health threat. In 2017, China experienced large-scale HFMD outbreaks of over 1.95 million cases with 96 fatalities (WHO Western Pacific). Besides China, HFMD outbreaks have been occurring periodically throughout the world in countries such as Taiwan, Japan, Vietnam, Singapore, and Malaysia. The main etiological agents for HFMD are EV-A71 and CV-A16. Currently, there are three licensed formaldehyde-inactivated HFMD vaccines in China. However, these vaccines could only confer protection at $80 \%$ efficacy against severe HFMD in comparison to $95 \%$ efficacy against mild HFMD. In addition, the monovalent EV-A71 vaccine is not protective against HFMD caused by CV-A16. With the rising concern of HFMD outbreaks, there is a need for an effective bivalent vaccine against both EV-A71 and CV-A16.

\section{Bivalent Hand, Foot, and Mouth Disease Vaccine}

The SP70 epitope is the main neutralizing epitope in EV-A71. The epitope was discovered to elicit neutralizing antibodies and conferred protection against homologous and heterologous EVA71 strain challenges in neonatal mice (Foo et al., 2007a,b). The SP70 epitope lies in the GH loop of VP1 (aa208-222) situated at the surface of the viral capsid (Plevka et al., 2012; Lyu et al., 2015a,b). CV-A16 also possesses an amino acid segment corresponding to the SP70 epitope (Figure 4; Ren et al., 2013, 2015).

To develop a bivalent vaccine, Zhao et al. (2015) designed a chimeric EV-A71 Virus-Like Particle (ChiEV-A71 VLP). The SP70 peptide of EV-A71 was replaced with the corresponding SP70 sequence from CV-A16 in ChiEV-A71 VLP (Zhao et al., 2015). The VLP was found to elicit robust Th1/Th2-dependent
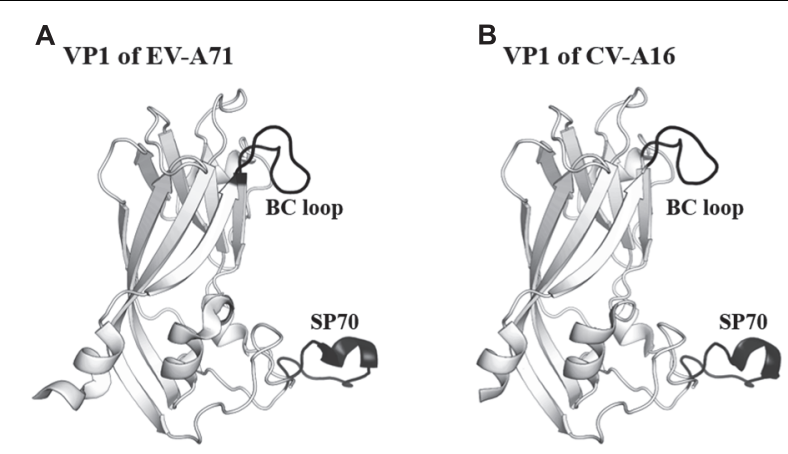

C

SP70 segment sequence alignment of EV-A71 and CV-A16

EV-A71 208-YPTFGEHKQEKDLEY-222

CV-A16 208-YPTFGEHLQANDLDY-222

FIGURE 4 | VP1 of EV-A71 and CV-A16. (A,B) Cartoon representation of VP1 (amino acids 76-276) of EV-A71 (PDB ID: 3VBS) and CV-A16 (PDB ID: $5 \mathrm{C} 4 \mathrm{~W})$. The $\mathrm{BC}$ loop and SP70 region are shown in black and labeled. (C) Sequence alignment of SP70 segments of EV-A71 and CV-A16. Amino acids that are not conserved between the two viruses are highlighted in light gray.

immune responses against EV-A71 and CV-A16. In addition, passive immunization with sera raised against ChiEV-A71 VLPs conferred full protection against lethal challenge with EV-A71 and CV-A16 in neonatal mice. Structural studies revealed that the SP70 epitope replacement converted the VLP surface charge potential from negative to neutral (Lyu et al., 2015a). The shift in the surface charge potential coupled with variations in amino acid sequences most likely accounted for the additional neutralization capability of the ChiEV-A71 VLP. Apart from EV-A71 and CV-A16, other coxsackieviruses such as CV-A6 and CV-A10 are emerging HFMD etiological agents (Chen et al., 2017; Anh et al., 2018). Incorporation of the neutralizing epitopes of each virus into a single VLP will enable the construction of a multivalent HFMD vaccine, provided the right approaches including structural vaccinology were utilized during vaccine design.

\section{Improvement of Vaccine Thermostability}

Despite being one of the most successful medical tools, there are still more than 17 million vaccine-preventable deaths caused by underutilization of vaccines (Clemens et al., 2010; Chen et al., 2011). This situation is especially true for the poorer countries, where cold chain is difficult to maintain, as most vaccines are sensitive to heat. The heat-unstable vaccines have poor efficacy when they are not properly refrigerated during delivery and storage. This led to incomplete immunizations during the implementation of vaccine programs (Schlehuber et al., 2011).

Self-biomineralization is one of the possible strategies to improve vaccine stability. Biomineralization is a mechanism where living organisms form exterior shells made of minerals such as calcium phosphate and calcium carbonate to cope with the harsh environments (Palmer et al., 2008; 
Farooq and Dietz, 2015). For instance, egg cells are enclosed by mineral shells to protect themselves from the external environment. Initially, research groups demonstrated that selfbiomineralization could be mimicked through the incorporation of self-biomineralizing peptides (Hartgerink et al., 2001; Lee et al., 2009). Subsequently, Wang et al. (2013) incorporated self-biomineralizing peptides into the BC loop of attenuated EV-A71 to improve the thermostability of the candidate vaccine (Wang et al., 2013). From 3D structures of EV-A71, they identified that the $\mathrm{BC}$ loop was externalized from the capsid and was suitable to be the region for peptide insertion (Figure 4). Importantly, the attenuated EV-A71 incorporated with selfbiomineralizing peptides displayed biomineralization capacity to form 5- to 10-nm exterior shells made of calcium phosphate. The vaccine exhibited enhanced thermostability, as it could be stored at $26^{\circ} \mathrm{C}$ for more than 9 days and at $37^{\circ} \mathrm{C}$ for 1 week. Intriguingly, they found that the biomineralized vaccine had improved immunogenicity, indicating an additional advantage of this approach.

\section{STRUCTURAL VACCINOLOGY PITFALLS}

Structural vaccinology has a great potential, but it faces some limitations. Firstly, this technology is susceptible to incomplete structural and immunological knowledge of the virus-induced host immune responses. For instance, mapping of a dengue epitope on available dengue structures revealed that the epitope was inaccessible in any of the known dengue configurations (Li et al., 2018). This indicated that the antibody recognized an as-yet unresolved conformation of the E protein displaying the epitope on the dengue surface. Another drawback is the static nature of X-ray crystallographic analysis of the antigen-antibody complexes. X-ray crystallography is one of the most utilized structural biology technique to understand antigen-antibody complexes (Toride and Brooks, 2018). However, the static picture provided by X-ray crystallography tends to obscure the role of structural plasticity in facilitating antigen-antibody interactions (Van Regenmortel, 2016). Generally, the structures visualized in antigen-antibody complexes and the free molecules differed at the binding sites due to structural alterations during binding. Therefore, the structure of an antigen-antibody complex determined using X-ray crystallography does not necessarily equate to the actual immunogenic structure recognized by the $\mathrm{B}$ cell receptors during antibody production. Intriguingly, it can be noted that most of the structural vaccinology efforts attempted to improve the B-cell antibody responses, but not the T-cell responses. This is problematic as the T-cell response is known to be associated with many antiviral properties including viral clearance and decreased viremia (Rosendahl et al., 2014). Furthermore, induction of T-cell response negated the effect of viral mutations, ultimately preventing them from escaping the immune responses (Rosendahl et al., 2014).

In addition to the aforementioned pitfalls, there are several review papers that have discussed the limitations of structural vaccinology to develop an effective vaccine against HIV (Pejchal and Wilson, 2010; Van Regenmortel, 2016). These pitfalls underscore the importance of acquiring new structural and functional insights into humoral immune responses against pathogens. Furthermore, incorporation of multiple structural techniques especially nuclear magnetic resonance (NMR) is favorable to study the structure and molecular dynamics of antigen-antibody complexes (Moraes et al., 2016). As structural vaccinology is still developing, its application is primarily limited to the design of recombinant protein vaccine, peptide vaccine, and VLPs. These vaccine platforms are associated with low immunogenicity. Therefore, it is crucial to integrate structural vaccinology with other complementary key technologies. For instance, DS-Cav1 protein vaccine could be integrated with the single-round replication recombinant vesicular stomatitis virus vector to potentially enhance its immunogenicity if required (Johnson et al., 2013). Lastly, a structural vaccinology approach should be extended to improve T-cell responses and not just focus on the $\mathrm{B}$-cell responses to produce better viral vaccines in the future.

\section{CONCLUSION}

Many of the structure-based vaccine candidates are under development, and some of them are in clinical trials (Robinson et al., 2015; Sastry et al., 2017; Huang et al., 2018). Recent advances have proven that structure-based vaccines are feasible. In some cases, structural knowledge is mandatory to circumvent the problems faced by conventional strategies. Based on current evidence, structural knowledge is essential to stabilize malleable antigens. It also allows the modifications of proteins or envelopes, either to enhance antigenicity or mask the non-neutralizing epitopes (Dey et al., 2009; de Taeye et al., 2018; Frei et al., 2018). Moreover, structure-based modifications are useful to lock antigenic proteins or envelopes in a certain conformation to maximize epitope presentation (McLellan et al., 2013a; Rutten et al., 2018). In addition, structural vaccinology can be utilized to design antibody therapies (Robinson et al., 2015; Huang et al., 2018). Lastly, a structural vaccinology approach can be used to improve vaccine thermostability, potentially solving the cold-chain problem faced in remote and poorer areas in large continents (Wang et al., 2013).

\section{AUTHOR CONTRIBUTIONS}

MIA wrote the manuscript. CLP provided the critical revision and final approval of the manuscript.

\section{FUNDING}

This work was funded by the Sunway University Research Centre Grant to the Centre for Virus and Vaccine Research (CVVR). 


\section{REFERENCES}

Anasir, M. I., Baxter, A. A., Poon, I. K. H., Hulett, M. D., and Kvansakul, M. (2017a). Structural and functional insight into Canarypox virus CNP058 mediated regulation of apoptosis. Viruses 9:305. doi: 10.3390/v9100305

Anasir, M. I., Caria, S., Skinner, M. A., and Kvansakul, M. (2017b). Structural basis of apoptosis inhibition by the fowlpox virus protein FPV039. J. Biol. Chem. 292, 9010-9021. doi: 10.1074/jbc.M116.768879

Anh, N. T., Nhu, L. N. T., Van, H. M. T., Hong, N. T. T., Thanh, T. T., Hang, V. T. T., et al. (2018). Emerging coxsackievirus A6 causing hand, foot and mouth disease, Vietnam. Emerg. Infect. Dis. 24, 654-662. doi: 10.3201/eid2404. 171298

Batra, G., Raut, R., Dahiya, S., Kamran, N., Swaminathan, S., and Khanna, N. (2010). Pichia pastoris-expressed dengue virus type 2 envelope domain III elicits virus-neutralizing antibodies. J. Virol. Methods 167, 10-16. doi: 10.1016/ j.jviromet.2010.03.002

Bhatt, S., Gething, P. W., Brady, O. J., Messina, J. P., Farlow, A. W., Moyes, C. L., et al. (2013). The global distribution and burden of dengue. Nature 496, 504-507. doi: 10.1038/nature 12060

Chen, L., Kwon, Y. D., Zhou, T., Wu, X., O’Dell, S., Cavacini, L., et al. (2009). Structural basis of immune evasion at the site of CD4 attachment on HIV-1 gp120. Science 326, 1123-1127. doi: 10.1126/science.1175868

Chen, M., He, S., Yan, Q., Xu, X., Wu, W., Ge, S., et al. (2017). Severe hand, foot and mouth disease associated with coxsackievirus A10 infections in Xiamen, China in 2015. J. Clin. Virol. 93, 20-24. doi: 10.1016/j.jcv.2017.05.011

Chen, X., Fernando, G. J., Crichton, M. L., Flaim, C., Yukiko, S. R., Fairmaid, E. J., et al. (2011). Improving the reach of vaccines to low-resource regions, with a needle-free vaccine delivery device and long-term thermostabilization. J. Control. Release 152, 349-355. doi: 10.1016/j.jconrel.2011. 02.026

Clemens, J., Holmgren, J., Kaufmann, S. H., and Mantovani, A. (2010). Ten years of the Global Alliance for vaccines and immunization: challenges and progress. Nat. Immunol. 11, 1069-1072. doi: 10.1038/ni1210-1069

Cockburn, J. J., Navarro Sanchez, M. E., Fretes, N., Urvoas, A., Staropoli, I., Kikuti, C. M., et al. (2012). Mechanism of dengue virus broad cross-neutralization by a monoclonal antibody. Structure 20, 303-314. doi: 10.1016/j.str.2012.01.001

Conibear, A. C., Rosengren, K. J., Daly, N. L., Henriques, S. T., and Craik, D. J. (2013). The cyclic cystine ladder in theta-defensins is important for structure and stability, but not antibacterial activity. J. Biol. Chem. 288, 10830-10840. doi: 10.1074/jbc.M113.451047

Corti, D., Voss, J., Gamblin, S. J., Codoni, G., Macagno, A., Jarrossay, D., et al. (2011). A neutralizing antibody selected from plasma cells that binds to group 1 and group 2 influenza A hemagglutinins. Science 333, 850-856. doi: 10.1126/ science. 1205669

Cox, A. L. (2015). MEDICINE. Global control of hepatitis C virus. Science 349, 790-791. doi: 10.1126/science.aad 1302

de Taeye, S. W., de la Pena, A. T., Vecchione, A., Scutigliani, E., Sliepen, K., Burger, J. A., et al. (2018). Stabilization of the gp120 V3 loop through hydrophobic interactions reduces the immunodominant V3-directed nonneutralizing response to HIV-1 envelope trimers. J. Biol. Chem. 293, 1688-1701. doi: $10.1074 /$ jbc.RA117.000709

de Taeye, S. W., Ozorowski, G., Torrents de la Pena, A., Guttman, M., Julien, J. P., van den Kerkhof, T. L., et al. (2015). Immunogenicity of stabilized HIV-1 envelope trimers with reduced exposure of non-neutralizing epitopes. Cell 163, 1702-1715. doi: 10.1016/j.cell.2015.11.056

Deng, L., Zhong, L., Struble, E., Duan, H., Ma, L., Harman, C., et al. (2013). Structural evidence for a bifurcated mode of action in the antibody-mediated neutralization of hepatitis C virus. Proc. Natl. Acad. Sci. U.S.A. 110, 7418-7422. doi: $10.1073 /$ pnas. 1305306110

Dey, B., Svehla, K., Xu, L., Wycuff, D., Zhou, T., Voss, G., et al. (2009). Structurebased stabilization of HIV-1 gp120 enhances humoral immune responses to the induced co-receptor binding site. PLoS Pathog. 5:e1000445. doi: 10.1371/ journal.ppat.1000445

Farooq, M. A., and Dietz, K. J. (2015). Silicon as versatile player in plant and human biology: overlooked and poorly understood. Front. Plant Sci. 6:994. doi: 10.3389/fpls.2015.00994

Foo, D. G., Alonso, S., Chow, V. T., and Poh, C. L. (2007a). Passive protection against lethal Enterovirus 71 infection in newborn mice by neutralizing antibodies elicited by a synthetic peptide. Microbes Infect. 9, 1299-1306. doi: 10.1016/j.micinf.2007.06.002

Foo, D. G., Alonso, S., Phoon, M. C., Ramachandran, N. P., Chow, V. T., and Poh, C. L. (2007b). Identification of neutralizing linear epitopes from the VP1 capsid protein of Enterovirus 71 using synthetic peptides. Virus Res. 125, 61-68. doi: 10.1016/j.virusres.2006.12.005

Frei, J. C., Wirchnianski, A. S., Govero, J., Vergnolle, O., Dowd, K. A., Pierson, T. C., et al. (2018). Engineered dengue virus domain III proteins elicit crossneutralizing antibody responses in mice. J. Virol. 92:e01023-18. doi: 10.1128/ JVI.01023-18

Graham, B. S., and Sullivan, N. J. (2018). Emerging viral diseases from a vaccinology perspective: preparing for the next pandemic. Nat. Immunol. 19, 20-28. doi: 10.1038/s41590-017-0007-9

Guy, B., Briand, O., Lang, J., Saville, M., and Jackson, N. (2015). Development of the Sanofi Pasteur tetravalent dengue vaccine: one more step forward. Vaccine 33, 7100-7111. doi: 10.1016/j.vaccine.2015.09.108

Guzman, M. G., Alvarez, M., and Halstead, S. B. (2013). Secondary infection as a risk factor for dengue hemorrhagic fever/dengue shock syndrome: an historical perspective and role of antibody-dependent enhancement of infection. Arch. Virol. 158, 1445-1459. doi: 10.1007/s00705-013-1645-3

Hadinegoro, S. R., Arredondo-Garcia, J. L., Capeding, M. R., Deseda, C., Chotpitayasunondh, T., Dietze, R., et al. (2015). Efficacy and long-term safety of a dengue vaccine in regions of endemic disease. N. Engl. J. Med. 373, 1195-1206. doi: 10.1056/NEJMoa1506223

Hai, R., Krammer, F., Tan, G. S., Pica, N., Eggink, D., Maamary, J., et al. (2012). Influenza viruses expressing chimeric hemagglutinins: globular head and stalk domains derived from different subtypes. J. Virol. 86, 5774-5781. doi: 10.1128/ JVI.00137-12

Halstead, S. B. (2017). Dengvaxia sensitizes seronegatives to vaccine enhanced disease regardless of age. Vaccine 35, 6355-6358. doi: 10.1016/j.vaccine.2017. 09.089

Hartgerink, J. D., Beniash, E., and Stupp, S. I. (2001). Self-assembly and mineralization of peptide-amphiphile nanofibers. Science 294, 1684-1688. doi: $10.1126 /$ science. 1063187

He, W., Mullarkey, C. E., Duty, J. A., Moran, T. M., Palese, P., and Miller, M. S. (2015). Broadly neutralizing anti-influenza virus antibodies: enhancement of neutralizing potency in polyclonal mixtures and IgA backbones. J. Virol. 89, 3610-3618. doi: 10.1128/JVI.03099-14

Hermida, L., Bernardo, L., Martin, J., Alvarez, M., Prado, I., Lopez, C., et al. (2006). A recombinant fusion protein containing the domain III of the dengue2 envelope protein is immunogenic and protective in nonhuman primates. Vaccine 24, 3165-3171. doi: 10.1016/j.vaccine.2006.01.036

Huang, Y., Karuna, S., Carpp, L. N., Reeves, D., Pegu, A., Seaton, K., et al. (2018). Modeling cumulative overall prevention efficacy for the VRC01 phase 2b efficacy trials. Hum. Vaccin. Immunother. 14, 2116-2127. doi: 10.1080/ 21645515.2018.1462640

Impagliazzo, A., Milder, F., Kuipers, H., Wagner, M. V., Zhu, X., Hoffman, R. M., et al. (2015). A stable trimeric influenza hemagglutinin stem as a broadly protective immunogen. Science 349, 1301-1306. doi: 10.1126/science.aac7263

Johnson, J. E., McNeil, L. K., Megati, S., Witko, S. E., Roopchand, V. S., Obregon, J. H., et al. (2013). Non-propagating, recombinant vesicular stomatitis virus vectors encoding respiratory syncytial virus proteins generate potent humoral and cellular immunity against RSV and are protective in mice. Immunol. Lett. 150, 134-144. doi: 10.1016/j.imlet.2012.12.005

Joyce, M. G., Wheatley, A. K., Thomas, P. V., Chuang, G. Y., Soto, C., Bailer, R. T., et al. (2016). Vaccine-induced antibodies that neutralize group 1 and group 2 influenza A viruses. Cell 166, 609-623. doi: 10.1016/j.cell.2016.06.043

Kaufmann, B., Plevka, P., Kuhn, R. J., and Rossmann, M. G. (2010). Crystallization and preliminary X-ray diffraction analysis of West Nile virus. Acta Crystallogr. Sect. F Struct. Biol. Cryst. Commun. 66(Pt 5), 558-562. doi: 10.1107/ S1744309110009899

Keck, Z., Wang, W., Wang, Y., Lau, P., Carlsen, T. H., Prentoe, J., et al. (2013). Cooperativity in virus neutralization by human monoclonal antibodies to two adjacent regions located at the amino terminus of hepatitis $\mathrm{C}$ virus E2 glycoprotein. J. Virol. 87, 37-51. doi: 10.1128/JVI.01941-12

Khan, A. G., Whidby, J., Miller, M. T., Scarborough, H., Zatorski, A. V., Cygan, A., et al. (2014). Structure of the core ectodomain of the hepatitis $C$ virus envelope glycoprotein 2. Nature 509, 381-384. doi: 10.1038/nature13117 
Kirkpatrick, E., Qiu, X., Wilson, P. C., Bahl, J., and Krammer, F. (2018). The influenza virus hemagglutinin head evolves faster than the stalk domain. Sci. Rep. 8:10432. doi: 10.1038/s41598-018-28706-1

Kong, L., Giang, E., Nieusma, T., Kadam, R. U., Cogburn, K. E., Hua, Y., et al. (2013). Hepatitis C virus E2 envelope glycoprotein core structure. Science 342, 1090-1094. doi: 10.1126/science.1243876

Kong, L., Giang, E., Nieusma, T., Robbins, J. B., Deller, M. C., Stanfield, R. L., et al. (2012a). Structure of hepatitis $\mathrm{C}$ virus envelope glycoprotein E2 antigenic site 412 to 423 in complex with antibody AP33. J. Virol. 86, 13085-13088. doi: 10.1128/JVI.01939-12

Kong, L., Giang, E., Robbins, J. B., Stanfield, R. L., Burton, D. R., Wilson, I. A., et al. (2012b). Structural basis of hepatitis C virus neutralization by broadly neutralizing antibody HCV1. Proc. Natl. Acad. Sci. U.S.A. 109, 9499-9504. doi: $10.1073 /$ pnas. 1202924109

Kong, L., Kadam, R. U., Giang, E., Ruwona, T. B., Nieusma, T., Culhane, J. C., et al. (2015). Structure of hepatitis $C$ virus envelope glycoprotein E1 antigenic site 314-324 in complex with antibody IGH526. J. Mol. Biol. 427, 2617-2628. doi: $10.1016 /$ j.jmb.2015.06.012

Krammer, F., Hai, R., Yondola, M., Tan, G. S., Leyva-Grado, V. H., Ryder, A. B., et al. (2014). Assessment of influenza virus hemagglutinin stalk-based immunity in ferrets. J. Virol. 88, 3432-3442. doi: 10.1128/JVI.03004-13

Krammer, F., Pica, N., Hai, R., Margine, I., and Palese, P. (2013). Chimeric hemagglutinin influenza virus vaccine constructs elicit broadly protective stalkspecific antibodies. J. Virol. 87, 6542-6550. doi: 10.1128/JVI.00641-13

Krey, T., Meola, A., Keck, Z. Y., Damier-Piolle, L., Foung, S. K., and Rey, F. A. (2013). Structural basis of HCV neutralization by human monoclonal antibodies resistant to viral neutralization escape. PLoS Pathog. 9:e1003364. doi: 10.1371/journal.ppat.1003364

Kuhn, R. J., Zhang, W., Rossmann, M. G., Pletnev, S. V., Corver, J., Lenches, E., et al. (2002). Structure of dengue virus: implications for flavivirus organization, maturation, and fusion. Cell 108, 717-725. doi: 10.1016/S0092-8674(02)00660-8

Kwong, P. D., Wyatt, R., Robinson, J., Sweet, R. W., Sodroski, J., and Hendrickson, W.A. (1998). Structure of an HIV gp120 envelope glycoprotein in complex with the CD4 receptor and a neutralizing human antibody. Nature 393, 648-659. doi: $10.1038 / 31405$

Laursen, N. S., Friesen, R. H. E., Zhu, X., Jongeneelen, M., Blokland, S., Vermond, J., et al. (2018). Universal protection against influenza infection by a multidomain antibody to influenza hemagglutinin. Science 362, 598-602. doi: 10.1126/science.aaq0620

Lee, P. S., and Wilson, I. A. (2015). Structural characterization of viral epitopes recognized by broadly cross-reactive antibodies. Curr. Top. Microbiol. Immunol. 386, 323-341. doi: 10.1007/82_2014_413

Lee, Y. J., Yi, H., Kim, W. J., Kang, K., Yun, D. S., Strano, M. S., et al. (2009). Fabricating genetically engineered high-power lithium-ion batteries using multiple virus genes. Science 324, 1051-1055. doi: 10.1126/science.1171541

Li, J., Watterson, D., Chang, C. W., Che, X. Y., Li, X. Q., Ericsson, D. J., et al. (2018). Structural and functional characterization of a cross-reactive dengue virus neutralizing antibody that recognizes a cryptic epitope. Structure 26, 51-59.e4. doi: 10.1016/j.str.2017.11.017

Lyu, K., He, Y. L., Li, H. Y., and Chen, R. (2015a). Crystal structures of yeast-produced enterovirus 71 and enterovirus 71/coxsackievirus A16 chimeric virus-like particles provide the structural basis for novel vaccine design against hand-foot-and-mouth disease. J. Virol. 89, 6196-6208. doi: 10.1128/JVI.00422-15

Lyu, K., Wang, G. C., He, Y. L., Han, J. F., Ye, Q., Qin, C. F., et al. (2015b). Crystal structures of enterovirus 71 (EV71) recombinant virus particles provide insights into vaccine design. J. Biol. Chem. 290, 3198-3208. doi: 10.1074/jbc. M114.624536

Magro, M., Mas, V., Chappell, K., Vazquez, M., Cano, O., Luque, D., et al. (2012). Neutralizing antibodies against the preactive form of respiratory syncytial virus fusion protein offer unique possibilities for clinical intervention. Proc. Natl. Acad. Sci. U.S.A. 109, 3089-3094. doi: 10.1073/pnas.1115941109

Mallajosyula, V. V., Citron, M., Ferrara, F., Temperton, N. J., Liang, X., Flynn, J. A., et al. (2015). Hemagglutinin sequence conservation guided stem immunogen design from influenza A H3 subtype. Front. Immunol. 6:329. doi: 10.3389/ fimmu.2015.00329
Manns, M. P., Buti, M., Gane, E., Pawlotsky, J. M., Razavi, H., Terrault, N., et al. (2017). Hepatitis C virus infection. Nat. Rev. Dis. Primers 3:17006. doi: 10.1038/ nrdp. 2017.6

McGuire, A. T., Dreyer, A. M., Carbonetti, S., Lippy, A., Glenn, J., Scheid, J. F., et al. (2014). HIV antibodies. Antigen modification regulates competition of broad and narrow neutralizing HIV antibodies. Science 346, 1380-1383. doi: 10.1126/science. 1259206

McLellan, J. S., Chen, M., Joyce, M. G., Sastry, M., Stewart-Jones, G. B., Yang, Y., et al. (2013a). Structure-based design of a fusion glycoprotein vaccine for respiratory syncytial virus. Science 342, 592-598. doi: 10.1126/science.1243283

McLellan, J. S., Chen, M., Leung, S., Graepel, K. W., Du, X., Yang, Y., et al. (2013b). Structure of RSV fusion glycoprotein trimer bound to a prefusion-specific neutralizing antibody. Science 340, 1113-1117. doi: 10.1126/science.1234914

Meola, A., Tarr, A. W., England, P., Meredith, L. W., McClure, C. P., Foung, S. K., et al. (2015). Structural flexibility of a conserved antigenic region in hepatitis $\mathrm{C}$ virus glycoprotein $\mathrm{E} 2$ recognized by broadly neutralizing antibodies. J. Virol. 89, 2170-2181. doi: 10.1128/JVI.02190-14

Moghaddam, A., Olszewska, W., Wang, B., Tregoning, J. S., Helson, R., Sattentau, Q. J., et al. (2006). A potential molecular mechanism for hypersensitivity caused by formalin-inactivated vaccines. Nat. Med. 12, 905-907. doi: 10.1038/nm1456

Moraes, A. H., Simonelli, L., Pedotti, M., Almeida, F. C., Varani, L., and Valente, A. P. (2016). Antibody binding modulates conformational exchange in domain III of dengue virus E protein. J. Virol. 90, 1802-1811. doi: 10.1128/JVI.02314-15

Nachbagauer, R., Krammer, F., and Albrecht, R. A. (2018). A live-attenuated prime, inactivated boost vaccination strategy with chimeric hemagglutininbased universal influenza virus vaccines provides protection in ferrets: a confirmatory study. Vaccines 6:E47. doi: 10.3390/vaccines6030047

Ozorowski, G., Pallesen, J., de Val, N., Lyumkis, D., Cottrell, C. A., Torres, J. L., et al. (2017). Open and closed structures reveal allostery and pliability in the HIV-1 envelope spike. Nature 547, 360-363. doi: 10.1038/nature23010

Palmer, L. C., Newcomb, C. J., Kaltz, S. R., Spoerke, E. D., and Stupp, S. I. (2008). Biomimetic systems for hydroxyapatite mineralization inspired by bone and enamel. Chem. Rev. 108, 4754-4783. doi: 10.1021/cr8004422

Pantophlet, R., and Burton, D. R. (2006). GP120: target for neutralizing HIV-1 antibodies. Ann. Rev. Immunol. 24, 739-769. doi: 10.1146/annurev.immunol. 24.021605.090557

Pejchal, R., and Wilson, I. A. (2010). Structure-based vaccine design in HIV: blind men and the elephant? Curr. Pharm. Des. 16, 3744-3753. doi: 10.2174/ 138161210794079173

Pierce, B. G., Boucher, E. N., Piepenbrink, K. H., Ejemel, M., Rapp, C. A., Thomas, W. D., et al. (2017). Structure-based design of hepatitis C virus vaccines that elicit neutralizing antibody responses to a conserved epitope. J. Virol. 91:e01032-17. doi: 10.1128/JVI.01032-17

Plevka, P., Perera, R., Cardosa, J., Kuhn, R. J., and Rossmann, M. G. (2012). Crystal structure of human enterovirus 71. Science 336:1274. doi: 10.1126/science. 1218713

Ren, J., Wang, X., Hu, Z., Gao, Q., Sun, Y., Li, X., et al. (2013). Picornavirus uncoating intermediate captured in atomic detail. Nat. Commun. 4:1929. doi: $10.1038 /$ ncomms 2889

Ren, J., Wang, X., Zhu, L., Hu, Z., Gao, Q., Yang, P., et al. (2015). Structures of coxsackievirus A16 capsids with native antigenicity: implications for particle expansion, receptor binding, and immunogenicity. J. Virol. 89, 10500-10511. doi: 10.1128/JVI.01102-15

Robinson, L. N., Tharakaraman, K., Rowley, K. J., Costa, V. V., Chan, K. R., Wong, Y. H., et al. (2015). Structure-guided design of an anti-dengue antibody directed to a non-immunodominant epitope. Cell 162, 493-504. doi: 10.1016/j.cell.2015. 06.057

Rosendahl, H. S., van Beek, J., de Jonge, J., Luytjes, W., and van Baarle, D. (2014). T cell responses to viral infections-opportunities for peptide vaccination. Front. Immunol. 5:171. doi: 10.3389/fimmu.2014.00171

Rutten, L., Lai, Y. T., Blokland, S., Truan, D., Bisschop, I. J. M., Strokappe, N. M., et al. (2018). A universal approach to optimize the folding and stability of prefusion-closed HIV-1 envelope trimers. Cell Rep. 23, 584-595. doi: 10.1016/j. celrep.2018.03.061

Saejung, W., Fujiyama, K., Takasaki, T., Ito, M., Hori, K., Malasit, P., et al. (2007). Production of dengue 2 envelope domain III in plant using TMV-based vector system. Vaccine 25, 6646-6654. doi: 10.1016/j.vaccine.2007.06.029 
Sandomenico, A., Leonardi, A., Berisio, R., Sanguigno, L., Foca, G., Foca, A., et al. (2016). Generation and characterization of monoclonal antibodies against a cyclic variant of hepatitis C virus E2 epitope 412-422. J. Virol. 90, 3745-3759. doi: 10.1128/JVI.02397-15

Sastry, M., Zhang, B., Chen, M., Joyce, M. G., Kong, W. P., Chuang, G. Y., et al. (2017). Adjuvants and the vaccine response to the DS-Cav1-stabilized fusion glycoprotein of respiratory syncytial virus. PLoS One 12:e0186854. doi: 10.1371/ journal.pone.0186854

Sautto, G. A., Kirchenbaum, G. A., and Ross, T. M. (2018). Towards a universal influenza vaccine: different approaches for one goal. Virol. J. 15:17. doi: 10.1186/ s12985-017-0918-y

Schlehuber, L. D., McFadyen, I. J., Shu, Y., Carignan, J., Duprex, W. P., Forsyth, W. R., et al. (2011). Towards ambient temperature-stable vaccines: the identification of thermally stabilizing liquid formulations for measles virus using an innovative high-throughput infectivity assay. Vaccine 29, 5031-5039. doi: 10.1016/j.vaccine.2011.04.079

Shao, W., Li, X., Goraya, M. U., Wang, S., and Chen, J. L. (2017). Evolution of influenza A virus by mutation and re-assortment. Int. J. Mol. Sci. 18:E1650. doi: 10.3390/ijms18081650

Steff, A. M., Monroe, J., Friedrich, K., Chandramouli, S., Nguyen, T. L., Tian, S., et al. (2017). Pre-fusion RSV F strongly boosts pre-fusion specific neutralizing responses in cattle pre-exposed to bovine RSV. Nat. Commun. 8:1085. doi: 10.1038/s41467-017-01092-4

Tarr, A. W., Owsianka, A. M., Timms, J. M., McClure, C. P., Brown, R. J., Hickling, T. P., et al. (2006). Characterization of the hepatitis C virus E2 epitope defined by the broadly neutralizing monoclonal antibody AP33. Hepatology 43, 592-601. doi: 10.1002/hep.21088

Tharakaraman, K., Robinson, L. N., Hatas, A., Chen, Y. L., Siyue, L., Raguram, S., et al. (2013). Redesign of a cross-reactive antibody to dengue virus with broadspectrum activity and increased in vivo potency. Proc. Natl. Acad. Sci. U.S.A. 110, E1555-E1564. doi: 10.1073/pnas.1303645110

Thullier, P., Demangel, C., Bedouelle, H., Megret, F., Jouan, A., Deubel, V., et al. (2001). Mapping of a dengue virus neutralizing epitope critical for the infectivity of all serotypes: insight into the neutralization mechanism. J. Gen. Virol. 82(Pt 8), 1885-1892. doi: 10.1099/0022-1317-82-8-1885

Thullier, P., Lafaye, P., Megret, F., Deubel, V., Jouan, A., and Mazie, J. C. (1999). A recombinant Fab neutralizes dengue virus in vitro. J. Biotechnol. 69, 183-190. doi: 10.1016/S0168-1656(99)00037-1

Toride, K. M., and Brooks, C. L. (2018). Epitope mapping of antibody-antigen interactions with X-ray crystallography. Methods Mol. Biol. 1785, 13-27. doi: 10.1007/978-1-4939-7841-0_2
Van Regenmortel, M. H. (2016). Structure-based reverse vaccinology failed in the case of HIV because it disregarded accepted immunological theory. Int. J. Mol. Sci. 17:E1591. doi: 10.3390/ijms17091591

Wang, G., Cao, R. Y., Chen, R., Mo, L., Han, J. F., Wang, X., et al. (2013). Rational design of thermostable vaccines by engineered peptide-induced virus self-biomineralization under physiological conditions. Proc. Natl. Acad. Sci. U.S.A. 110, 7619-7624. doi: 10.1073/pnas.1300233110

Wang, H., Cohen, A. A., Galimidi, R. P., Gristick, H. B., Jensen, G. J., and Bjorkman, P. J. (2016). Cryo-EM structure of a CD4-bound open HIV-1 envelope trimer reveals structural rearrangements of the gp120 V1V2 loop. Proc. Natl. Acad. Sci. U.S.A. 113, E7151-E7158. doi: 10.1073/pnas.1615939113

Wu, N. C., Zost, S. J., Thompson, A. J., Oyen, D., Nycholat, C. M., McBride, R., et al. (2017). A structural explanation for the low effectiveness of the seasonal influenza H3N2 vaccine. PLoS Pathog. 13:e1006682. doi: 10.1371/journal.ppat. 1006682

Wu, X., Yang, Z. Y., Li, Y., Hogerkorp, C. M., Schief, W. R., Seaman, M. S., et al. (2010). Rational design of envelope identifies broadly neutralizing human monoclonal antibodies to HIV-1. Science 329, 856-861. doi: 10.1126/science. 1187659

Zhang, Z. S., Yan, Y. S., Weng, Y. W., Huang, H. L., Li, S. Q., He, S., et al. (2007). High-level expression of recombinant dengue virus type 2 envelope domain III protein and induction of neutralizing antibodies in BALB/C mice. J. Virol. Methods 143, 125-131. doi: 10.1016/j.jviromet.2007.02.012

Zhao, H., Li, H. Y., Han, J. F., Deng, Y. Q., Zhu, S. Y., Li, X. F., et al. (2015). Novel recombinant chimeric virus-like particle is immunogenic and protective against both enterovirus 71 and coxsackievirus A16 in mice. Sci. Rep. 5:7878. doi: 10.1038/srep07878

Zhou, T., Georgiev, I., Wu, X., Yang, Z. Y., Dai, K., Finzi, A., et al. (2010). Structural basis for broad and potent neutralization of HIV-1 by antibody VRC01. Science 329, 811-817. doi: 10.1126/science.1192819

Conflict of Interest Statement: The authors declare that the research was conducted in the absence of any commercial or financial relationships that could be construed as a potential conflict of interest.

Copyright (C) 2019 Anasir and Poh. This is an open-access article distributed under the terms of the Creative Commons Attribution License (CC BY). The use, distribution or reproduction in other forums is permitted, provided the original author(s) and the copyright owner(s) are credited and that the original publication in this journal is cited, in accordance with accepted academic practice. No use, distribution or reproduction is permitted which does not comply with these terms. 